\title{
Semantic and morphological characteristics of a noun as a subject in the modern French language
}

\section{[Les caractéristiques sémantiques et morphologiques d'un nom comme sujet en français contemporain]}

\author{
Aleksei A. Zaitsev - Dinara G. Vasbieva
}

DOI: 10.18355/XL.2021.14.04.15

\begin{abstract}
The present study considers the problem of the relationship between semantics and morphology of nouns in the syntactic function of the subject in the modern French literary language. This work aims to answer the questions of whether the syntactic function is not indifferent to the semantic nature of the noun and whether any noun can be used in the function of the subject. The text fragments taken from the works of classic French writers Honore de Balzac, Emile Zola, André Maurois, François Moryac, etc. have been investigated. The total length of the analyzed texts taken in equal portions of 100 phrases per work was 85,000 tokens. The general methodological principles of this study are the provisions on the dialectical connection between language and thinking, on the one hand, and language and reality, on the other. The findings show that the choice of grammatical forms of a noun in these syntactic functions is not random and it is determined by the patterns of thought formation, which reflects the objective situation.
\end{abstract}

Key words: noun, semantics, morphology, subject, modern French language

\section{Résumé}

L'article est consacré au problème de l'interaction de la sémantique et de la morphologie du nom en fonction syntaxique du sujet dans la langue littéraire française contemporaine. Le travail répond à la question de savoir si la fonction syntaxique est indifférente à la nature sémantique du nom, si tout nom peut être utilisé en fonction du sujet. L'étude a été menée sur le matériel des textes tirés des œuvres des écrivains classiques français Honoré de Balzac, Émile Zola, Antoine de Saint-Exupéry, André Maurois, François Mauriac etc. La longueur totale des textes analysés, pris en portions égales de 100 phrases de chaque œuvre, était de 85000 mots. Les principes méthodologiques généraux de cette étude sont les conceptions sur le lien dialectique du langage et de la pensée, d'une part, et du langage et de la réalité, d'autre part. En général, les résultats de l'étude montrent que le choix des formes grammaticales d'un nom en ces fonctions syntaxiques n'est pas aléatoire, il est déterminé par les modèles de la conception de la pensée, ce qui reflète une situation objective.

Mot clés: le nom, la sémantique, la morphologie, le sujet, le français contemporain

\section{Introduction}

La structure grammaticale d'une langue est étroitement liée à ses autres aspects, et surtout à celui lexico-sémantique. La relation entre la grammaire et le vocabulaire qui se manifeste principalement par diverses formes de restrictions lexicales imposées aux modèles grammaticaux par rapport à la langue française n'a pas encore été suffisamment étudiée.

En effet, comme Lapteva (1976) l'a souligné, «évidemment, il y a d'autres formes d'influence sur la grammaire. Les processus qui recouvrent la structure grammaticale de la langue littéraire en relation avec les phénomènes de renforcement de sa délimitation fonctionnelle propres à toute langue littéraire développée, n'ont pas

XLinguae, Volume 14 Issue 4, October 2021, ISSN 1337-8384, eISSN 2453-711X 
encore été étudiés». Dans ses recherches, Combettes (2012: 27) écrit qu' «il est évident que la situation est tout à fait différente en français moderne: l'obligation de l'expression du sujet et la fixation de l'ordre des mots obligent à identifier le SN comme sujet syntaxique, du moins dans la phrase «liée» «SN $+\mathrm{SV} »$.

Le cœur du problème est le suivant. Dans toute grammaire, il est dit, par exemple, que dans une phrase, le sujet peut généralement être exprimé par un nom. Mais tout nom peut-il être utilisé dans cette fonction? La question se pose de la relation entre la signification du nom et ses fonctions syntaxiques dans la phrase, c'est-à-dire la question de savoir si la fonction syntaxique n'est pas indifférente à la nature sémantique du nom.

Le nom ou le substantif est la principale partie dénominative du discours. Il est caractérisé par les trois aspects suivants: valeur sémantique, formes (catégories grammaticales), fonctions syntaxiques.

Dans l'aspect sémantique la notion d'objet exprimée par le nom est une abstraction notionnelle qui embrasse les mots désignant les êtres animés (chien, enfant), les objets inanimés (tapis, maison), les actions (départ), les sentiments (joie), les notions abstraites (justice), la matière (fer, viande).

De nombreux chercheurs partagent l'opinion selon laquelle le nom français est caractérisé par les catégories grammaticales suivantes: genre, nombre, détermination - indétermination (Gak, 2000; Vassilieva, 2010; Etheves, 2016; Babushkina \& Kalugina, 2020). Les catégories grammaticales sont toujours relatives à une langue donnée, et à une certaine période de l'histoire de chaque langue. Le plus souvent on désigne sous le nom de catégories grammaticales les notions qui s'expriment au moyen des morphèmes. Dans quelque langue qu'on les considère, les catégories grammaticales ne se définissent que par la forme qui les exprime.

L'histoire des catégories grammaticales peut souvent dans chaque langue s'établir avec exactitude. Mais le système des catégories apparaît suivant les langues sous des formes assez différentes. La classification des catégories grammaticales est un travail de morphologie générale qui reste encore à faire (Baugh, Cable, 2012).

Dans la proposition le substantif acquiert une fonction de terme de proposition, ses fonctions sont nombreuses. Le nom peut servir de sujet (S), complément direct et indirect (C), complément d'agent, attribut, complément attributif d'un autre nom, complément circonstanciel, complément prédicatif.

L'étude du problème de la relation entre la signification du nom et ses fonctions dans la phrase est d'autant plus importante que l'indicateur d'interaction que nous ne pouvons extraire que des données concrètes de sa mise en œuvre dans la parole, est également un signe qualitatif du langage en tant que système. Le fait que la structure formelle et sémantique d'une phrase ne coïncide pas toujours a conduit certains linguistes à penser qu'il n'y a pas de lien nécessaire entre la forme grammaticale d'une phrase et son contenu lexical et que, par conséquent, «une description d'une langue combinant des déclarations identiques dans le sens mais différentes dans la forme n'aurait aucune valeur scientifique» (Martinet, 1978: 197).

Ce point de vue est caractéristique non seulement de certains domaines de la linguistique dite structurelle (principalement américaine), mais aussi de nombreuses grammaires descriptives de différentes langues, classant les unités syntaxiques en fonction de leurs caractéristiques formelles externes. Ainsi, dans la «Grammaire de la langue russe» académique, on peut lire que «le contenu concret des phrases ne peut pas faire l'objet d'une considération grammaticale» (Shvedova, 1980).

Le problème de la relation entre les caractéristiques sémantiques et morphologiques d'un nom et sa fonction syntaxique a été posé sous une forme générale dans les travaux de linguistes français et russes, mais il a certainement besoin d'une analyse plus profonde sur des matériaux concrets de la parole, puisque l'on ne voit l'interaction de diverses catégories que dans les actes de langage. 


\section{Révision de la littérature}

Il est à signaler qu'à l'heure actuelle, en linguistique russe et étrangère, il existe des travaux où les problèmes d'interaction du vocabulaire et de la grammaire sont résolus d'une nouvelle manière. Dans ces travaux, lors de la classification des unités syntaxiques (types de proposition, termes de proposition), les significations catégorielles des lexèmes sont prises en compte (animation-inanimation, abstraitconcret, etc.). Des expériences intéressantes sur la modélisation des phrases de base ont été menées par les psycholinguistes français J. Dubois et L. Irigaray. Ces expériences ont montré que la fonction syntaxique n'est pas indifférente à la nature sémantique du nom, de plus, elle en dépend. «La loi de la correspondance sémantique-syntaxique, plus obligatoire pour les noms animés que pour les inanimés, agit comme l'une des conditions fondamentales de la formation de phrases élémentaires», concluent les auteurs des expériences (Dubois, Irigaray, 1966). «Puisque la structure sémantique de la phrase se réfère principalement aux phénomènes de la langue, et non aux faits mentaux, sociaux ou physiques, ses éléments peuvent être définis comme des unités du plan sémantique seulement par leur relation avec le plan de l'expression formelle-linguistique» - affirme A. Greimas (Greimas, 1996: 172).

Dans ses travaux G. Gak indique que la fonction syntaxique du sujet n'est pas tout à fait formelle et dépourvue du contenu (Gak, 2004). Ainsi, si le sujet désigne un sujet effectuant une certaine action active, alors tous les noms ne peuvent pas être sujets. Tout d'abord une personne animée peut remplir ce rôle. T. B. Alisova a consacré sa recherche à l'explication des relations entre les unités grammaticales formelles et leur contenu. Dans un travail intéressant «Essais de syntaxe de la langue italienne contemporaine», elle souligne que «le remplissage lexical de la position $\mathrm{du}$ sujet grammatical a un certain nombre de limites qui dépendent de la relation sémantique-communicative entre le sujet et le prédicat» (Alissova, 2009).

L'aspect sémantique d'un nom se manifeste à la fois dans sa propre signification (animation, inanimation, abstraction, concrétisation, etc.) et dans ses catégories morphologiques significatives.

Si la relation de la fonction syntaxique du nom avec sa propre sémantique a été considérée dans les travaux ci-dessus, la relation de cette fonction avec la catégorie morphologique du nombre, par exemple, n'a presque pas été étudiée.

Ce travail est consacré à l'analyse de l'interaction de la sémantique et de la morphologie du nom dans la fonction syntaxique du sujet, tout en tenant compte de la caractéristique du nom dans la fonction du complément direct et indirect et de la caractéristique du prédicat.

\section{Matériaux et Méthodes}

Pour établir des relations quantitatives entre les formes et les fonctions des constituants nominaux d'une phrase simple en français, des textes issus des œuvres littéraires des écrivains français classiques et contemporains ont été analysés (Honoré de Balzac, Émile Zola, Antoine de Saint-Exupéry, André Maurois, François Mauriac etc.). De chaque œuvre 100 phrases ont été prises de suite. Puisque tout d'abord nous nous intéressions au sujet exprimé par le nom dans une phrase simple, alors l'analyse n'a pas pris en compte les phrases sans sujet et sans verbe, ainsi que les phrases dans le dialogue et les phrases introduisant un discours direct. Les textes ont été décomposés en phrases simples, c'est-à-dire des constructions prédicatives dont les constituants sont reliés par une connexion syntaxique avec la présence obligatoire du sujet exprimé par un nom ou un pronom.

Ainsi, les phrases complexes ont été analysées comme deux phrases simples. Les phrases avec des termes homogènes (dans la fonction du prédicat) étaient considérées comme plusieurs phrases simples avec le même sujet. Les phrases avec

XLinguae, Volume 14 Issue 4, October 2021, ISSN 1337-8384, eISSN 2453-711X 
gérondif étaient classées de la même manière. Lors de la répétition de la même phrase elle n'a été prise en compte qu'une seule fois.

Pour collecter des données empiriques, une analyse théorique de la littérature relative au sujet de recherche, linguistique, philosophique et psychologique, a été appliquée. Pour le traitement des données empiriques, il a été fait usage de méthodes de modélisation linguistique, ainsi que d'un traitement de données statistiques, l'interprétation de celles-ci étant effectuée par des méthodes de recherche comparative, transformationnelle et descriptive.

La recherche a été menée en trois étapes.

Au cours de la première étape, le choix de l'objet, du sujet et du problème de recherche, ainsi que l'analyse théorique, ont été effectués ; la base de recherche et la méthodologie ont été déterminées, l'hypothèse a été formulée.

Lors de la deuxième étape, nous avons révélé par la méthode de l'échantillonnage continu des phrases contenant le nom en fonction du sujet. Les exemples donnés en guise d'illustration sont très loin d'épuiser le corpus étudié.

À la troisième étape, nous avons procédé à l'analyse et la synthèse des résultats de la recherche linguistique et proposé le modèle la relation entre la signification du nom et ses fonctions dans la phrase.

\section{Résultats et discussion}

Dans ce travail, nous avons examiné la catégorie morphologique du nombre d'un nom en français et la catégorie de la détermination qui est sémantiquement étroitement liée à la catégorie du nombre et s'exprime par l'article qui accompagne le nom dans la phrase. En même temps, nous avons retiré de l'analyse la catégorie morphologique du genre des noms, puisque cette catégorie n'est significative que pour les noms animés où elle reflète les différences de genre. Pour les noms inanimés (et il y en a une majorité absolue dans la langue) la catégorie de genre est une forme pure.

La catégorie du nombres chez les noms en français est significative pour les noms concrets dénombrables, animés ou inanimés. La notion du nombre suppose, en premier lieu, une opposition de la singularité à la pluralité. Cette opposition est réalisée dans le discours à l'aide des indicateurs morphologiques de valeurs numériques (modification du son des extrémités des mots, différentes fins, etc.).

En cas de l'indifférenciation morphologique du nom, la catégorie du nombre est identifiée par la forme des articles (exprimée par l'opposition des articles le, la / les; un, une / des ).

En outre, la catégorie du nombre est adjacente à la catégorie de la totalité/partialité (chez les noms anumériques), exprimée par l'opposition des articles le, la / du, de la.

En plus de la fonction morphologique (précision du nombre et du genre des noms), l'article remplit également une fonction sémantique-syntaxique - une expression de détermination. La possibilité ou l'impossibilité du choix est un critère du concept de détermination. Dans le discours, cette catégorie est représentée par l'opposition des articles définis et indéfinis le, la / un, une. La notion de détermination des noms s'exprime aussi à l'aide de certains déterminants mon, ma, mes, ton, ta, tes, son, sa, ses, ce, cette, ces, l'autre, les autres, tous les les deux, trois, etc. et des noms au déterminatif indéfini un autre, d'autres, tout, chaque, divers, aucun, plusieurs, quelques, maints, deux, trois, quatre, etc. exprimant la notion d'in détermination.

Dans la phrase, en plus des noms, les pronoms, les termes substantivés, les infinitifs, les propositions subordonnées peuvent agir comme des termes nominaux. Dans ce cas au cours de l'analyse le nom correspondant à cette fonction a été restauré par substitution (si possible). Ainsi, les pronoms personnels et relatifs, par exemple, qui étaient utilisés en fonction des sujets, des compléments (directs ou indirects) ou des attributs, ont été remplacés par des noms avec lesquels ces pronoms étaient corrélés dans le contexte. Le sujet ou le complément exprimé par les noms-termes 
homogènes de la phrase était considéré comme un simple pluriel défini ou indéterminé. Si le complément a été formalisé par un article ou un déterminant, il a été classé comme un élément distinct quel que soit le degré de sa fusion sémantique avec le verbe. Le complément non formalisé par l'article ou le déterminatif était considéré comme faisant partie du groupe verbal et toute la construction de ce type a été identifiée comme un seul verbe analytique.

En l'absence de l'article défini dans le cas de la neutralisation de la forme indéterminée ou partielle d'articles (après la préposition de, lors de de la négation, etc.) la forme de l'article était reprise par la transformation de cette structure, en tenant compte, naturellement, de la sémantique du nom. Parmi les prédicats différents: I) le prédicat exprimé par le verbe intransitif: a) sans complément d'objet, b) avec complément d'objet indirect ou circonstanciel; 2) le prédicat de transition: a) avec complément d'objet direct, b) sans complément d'objet; 3) prédicat nominal: tous les attributs ont été répartis en trois groupes: a) la combinaison du verbe être + nom sans article ou déterminatif a été considérée comme un groupe verbal, c'est-à-dire que ce nom était inclus dans le groupe du verbe, b) la combinaison du verbe être + nom avec l'article défini, c) être + nom avec un article indéfini. Le prédicat verbal complexe a été classé par infinitif.

Au cours de l'analyse des textes on a révélé les structures suivantes avec des caractéristiques de fréquence correspondantes (pour 500 phrases)

1. Le complément d'objet est absent ou bien n'est pas exprimé par un nom:

a) $S$ le $(l a)+V-82$ exemples

Où, sans doute, grondait l'ascension de la mer (Antoine de Saint-Exupéry, 2018); Cette illusre femme resta victorieuse (Honoré de Balzac, 2012).

b) $S$ les $+V-26$ exemples

Si les montagnent ont dérivé, si... (Antoine de Saint-Exupéry, 2018); Les hommes de la famille vont arriver (François Mauriac, 2007).

c) $S$ des $+V-6$ exemples

Des trombes marines se dressaient là .... (Antoine de Saint-Exupéry, 2018); Des renseignements peuvent se reduire à ceci (Honoré de Balzac, 2017).

d) $S$ un (une) $+V-4$ exemples

D'où sortait une voix flûtée d'enfant ... (Émile Zola, 2002); Un silence se fit (Antoine de Saint-Exupéry, 2018).

2. Le complément d'objet direct ou indirect est exprimé par un attribut avec un article ou un déterminant, une circonstance:

a) S le (la $)+V+C-268$ exemples

Quand le sable contenqit un petit caillou ... (André Maurois, 2014); Le mécanicien poite la carte ... (Antoine de Saint-Exupéry, 2018).

b) $S$ les $+V+C-81$ exemples

Mais les moteurs remplissent cette chambre éclairée d'un frémissement (Antoine de Saint-Exupéry, 2018); Où les femmes ne voient de pitié ... (Honoré de Balzac, 2012).

c) $S$ des $+V+C-17$ exemples

Des cigarettes ponctuaient des méditations .... (Antoine de Saint-Exupéry, 2018); Des passants tournaient la tête ... (Émile Zola, 2017).

d) $S$ un (une) $+V+C-16$ exemples

Un corset le serrait à étouffer ... (François Mauriac, 2009); Mais une nouvelle rencontre l'arrêta (Émile Zola, 2002). 


\section{Tableau 1}

\begin{tabular}{|c|c|c|c|c|c|c|c|c|}
\hline \multirow{2}{*}{$\begin{array}{c}\text { Suje } \\
\mathrm{t}\end{array}$} & \multicolumn{5}{|c|}{ Complément d'objet } & \multirow{2}{*}{$\sum$} & \multirow{2}{*}{$\begin{array}{c}\text { Absence } \\
\text { du } \\
\text { complé } \\
\text { ment } \\
\text { d'objet }\end{array}$} & \multirow{2}{*}{$\Sigma$} \\
\hline & le, la & $\begin{array}{l}\text { un, } \\
\text { une }\end{array}$ & les & des & $\begin{array}{c}\mathrm{du}, \mathrm{de} \\
\text { la }\end{array}$ & & & \\
\hline le, la & $\frac{75 / 77}{152}$ & $\frac{18 / 2}{\frac{7}{45}}$ & $\frac{22 / 2}{\frac{8}{50}}$ & $\frac{6 / 7}{13}$ & $\frac{3 / 5}{8}$ & $\frac{124 / 144}{268}$ & $\frac{47 / 35}{82}$ & $\frac{171 / 179}{350}$ \\
\hline $\begin{array}{l}\text { un, } \\
\text { une }\end{array}$ & $\frac{10 / 5}{15}$ & - & $\frac{1 / 0}{1}$ & - & - & $\frac{11 / 5}{16}$ & $\frac{4 / 0}{4}$ & $\frac{15 / 5}{20}$ \\
\hline les & $\frac{12 / 24}{36}$ & $\frac{9 / 7}{16}$ & $\frac{9 / 9}{18}$ & $\frac{2 / 6}{8}$ & $\frac{1 / 2}{3}$ & $\frac{33 / 48}{81}$ & $\frac{17 / 9}{26}$ & $\frac{50 / 57}{107}$ \\
\hline des & $\frac{5 / 0}{5}$ & $\frac{0 / 2}{2}$ & $\frac{4 / 2}{6}$ & $\frac{3 / 1}{4}$ & - & $\frac{12 / 5}{17}$ & $\frac{4 / 2}{6}$ & $\frac{16 / 7}{23}$ \\
\hline$\sum$ & $\frac{102 / 10}{\underline{6}}$ & $\frac{27 / 3}{\frac{6}{63}}$ & $\frac{36 / 3}{\frac{9}{75}}$ & $\frac{11 / 14}{25}$ & $\frac{4 / 7}{11}$ & $\frac{180 / 202}{382}$ & $\frac{72 / 46}{118}$ & $\frac{252 / 248}{500}$ \\
\hline
\end{tabular}

Dans les tableaux, les chiffres à gauche de la barre oblique indiquent le nombre de noms, à droite - le nombre de pronoms en fonction du sujet, sous la barre - le nombre total.

En nous basant sur le tableau 1, nous pouvons déterminer la relation quantitative (non absolue) entre les formes et les fonctions des termes nominaux d'une phrase simple (d'après le nombre d'exemples étudiés).

En poursuivant la délimitation des concepts de nombre et de détermination en fonction du sujet, nous avons obtenu les données suivantes:

Tableau 2

\begin{tabular}{|c|c|c|c|c|c|}
\hline \multirow{2}{*}{ Sujet } & \multicolumn{2}{|c|}{ Complément d'objet } & \multirow{2}{*}{$\sum$} & \multirow{2}{*}{$\begin{array}{l}\text { Absence du } \\
\text { complément } \\
\text { d'objet }\end{array}$} & \multirow{2}{*}{$\sum$} \\
\hline & singulier & pluriel & & & \\
\hline \multirow{2}{*}{ singulier } & $103 / 109$ & $\underline{32 / 40}$ & $135 / 149$ & $\underline{51 / 35}$ & $\underline{186 / 184}$ \\
\hline & 212 & 72 & 284 & 86 & 370 \\
\hline \multirow{2}{*}{ pluriel } & $26 / 33$ & $\underline{19 / 20}$ & $45 / 53$ & $21 / 11$ & $\underline{66 / 64}$ \\
\hline & 59 & 39 & 98 & 32 & 130 \\
\hline \multirow[b]{2}{*}{$\sum$} & $\underline{129 / 142}$ & $\underline{51 / 60}$ & $\underline{180 / 202}$ & $\underline{72 / 46}$ & $\underline{252 / 248}$ \\
\hline & 271 & 111 & 382 & 118 & 500 \\
\hline
\end{tabular}

Tableau 3

\begin{tabular}{|c|c|c|c|c|c|}
\hline \multirow{2}{*}{ Sujet } & \multicolumn{2}{|c|}{ Complément d'objet } & \multirow{2}{*}{$\sum$} & \multirow{2}{*}{$\begin{array}{c}\text { Absence du } \\
\text { complément } \\
\text { d'objet }\end{array}$} & \multirow{2}{*}{$\sum$} \\
\hline & défini & indéfini & & & \\
\hline \multirow{2}{*}{ défini } & $\underline{118 / 138}$ & $\underline{39 / 54}$ & $\underline{157 / 192}$ & $\underline{64 / 44}$ & $221 / 236$ \\
\hline & 256 & 93 & 349 & 108 & 457 \\
\hline \multirow{2}{*}{ indéfini } & $\underline{20 / 7}$ & $\underline{3 / 3}$ & $\underline{23 / 10}$ & $\underline{8 / 2}$ & $\underline{31 / 12}$ \\
\hline & $\overline{27}$ & $\overline{6}$ & 33 & 10 & 43 \\
\hline \multirow{2}{*}{$\Sigma$} & $\underline{138 / 145}$ & $\underline{42 / 57}$ & $\underline{180 / 202}$ & $\underline{73 / 46}$ & $\underline{252 / 248}$ \\
\hline & 283 & 99 & 382 & $\overline{118}$ & 500 \\
\hline
\end{tabular}


Comme on peut le voir dans les tableaux ci-dessus, la fonction syntaxique du sujet est dominée par les noms au singulier - 370 fois ou $74 \%$, y compris en l'absence du terme verbal $-17,2 \%$, avec le terme verbal - 50,8 \%. Dans cette fonction, le nom au pluriel dans cette fonction apparaissait 130 fois ou $26 \%$ (respectivement, 6,4\%, 19,6\%).

Dans les exemples étudiés, il existe également une prédominance absolue de l'utilisation en fonction du sujet des noms avec un déterminatif défini - 91,4\%, y compris en l'absence du complément - 21,6\%, avec complément - 69,8\%. Les noms avec un déterminatif indéfini dans cette fonction n'ont été rencontrés que 43 fois ou $8,6 \%$ (respectivement $2 \%, 6,6 \%$ ).

Il serait intéressant de considérer la relation entre le sujet et le complément d'objet du point de vue de la distribution des indicateurs des catégories considérées. Dans la catégorie du nombre, comme dans la catégorie de la détermination, il existe théoriquement 4 types de structures, qui dans les exemples étudiés, en fonction de la fréquence d'utilisation se sont répartis comme suit:

I. Dans la catégorie du nombre: $S s g+V+C s g-55,5 \%$; $S s g+V+C p l-$ $18,9 \% ; S p l+V+C s g-15,4 \% ; S p l+V+C p l-10,2 \%$.

II. Dans la catégorie de la de la détermination: $S l e+V+C l e-67 \%$; $S l e+V$ $+C$ un - 24,4\%; $S$ un $+V+C l e-7,1 \% ; S$ un $+V+C$ un $-1,5 \%$.

Il est à signaler que les formes marquées $(\mathrm{M})$ et non marquées $(\mathrm{N})$ occupent dans les types de ces structures la même place du point de vue de leur répartition par fréquence:

La catégorie du nombre:

La catégorie de la détermination:
I. $\quad \mathrm{N}-\mathrm{N}$
I. $\quad \mathrm{N}-\mathrm{N}$
II. $\mathrm{N}-\mathrm{M}$
II. $\mathrm{N}-\mathrm{M}$
III. $\mathrm{M}-\mathrm{N}$
III. $\mathrm{M}-\mathrm{N}$
IV. $\mathrm{M}-\mathrm{M}$
VI. $\mathrm{M}-\mathrm{M}$

Les résultats de la recherche montrent que la fonction syntaxique du sujet est liée en premier lieu aux catégories de la détermination et du singulier. Parmi les types de structures prédominent le plus souvent celles où le sujet et le complément d'objet sont exprimés par des noms avec un déterminatif défini au singulier.

Beaucoup moins souvent nous trouvons des constructions où le sujet ou le complément d'objet sont exprimés par un nom sous une forme marquée et extrêmement rarement des types de structures avec un nom au pluriel indéfini en fonction de sujet et de complément d'objet.

Bien entendu, une telle relation entre les formes morphologiques et les fonctions du nom n'est pas aléatoire. Parmi les facteurs qui le conditionnent, nous pouvons noter les suivants. Ainsi, en raison de l'asymétrie du signe linguistique la même forme peut exprimer des significations différentes, alors que la même signification peut être exprimée par de différentes formes. Ainsi, les formes du nombre sont sémantiquement hétérogènes. La forme singulière, par exemple, peut exprimer l'unité chez les objets dénombrables, la généralisation, peut être insignifiante chez les noms anumériques. De même, les formes d'un article défini et d'un article indéfini sont également polysémiques. L'article défini peut exprimer non seulement la détermination, contrairement à l'indétermination, mais aussi remplir une fonction généralisante dans laquelle l'opposition de la certitude à l'indétermination est supprimée. La forme singulière et la forme de l'article défini sont, comme on a déjà noté ci-dessus, des formes non marquées dans le système de formes de ces catégories. 
Cela explique, en partie, un grand emploi de ces formes chez les noms dans n'importe quelle fonction, y compris en fonction de sujet.

Quant à la distribution des formes de nombres, nous pouvons comparer ici nos observations avec les conclusions de J. Dubois qui estime également qu'en français contemporain, malgré le grand nombre de noms utilisés dans le discours au pluriel,en tant que sujet le nom est généralement employé au singulier (Dubois, 1967). Pour faire cela, on recourt même à la transformation de la structure active en une structure passive et vice versa. Certes, J. Dubois explique ce phénomène par la loi d'économie du langage (le sujet et donc le prédicat sont au singulier, ce qui est plus économique pour écrit, par exemple).

Cependant, à notre avis, ce phénomène doit être abordé du point de vue de l'organisation de la pensée, dont le processus implique le passage du plus simple au plus complexe. Par conséquent, dans le processus de parler, si possible, on passe d'un simple du singulier au pluriel plus complexe. La tendance à l'emploi du type structurel $S l e+V+C$ un est sans aucun doute également liée au fait qu'il correspond à l'habituelle division effective de la phrase, dans laquelle le message passe du donné (exprimé par un déterminatif défini) au nouveau (indiqué par un déterminatif indéfini). En ce qui concerne l'ordre direct des mots en français, le donné s'exprime généralement par la forme syntaxique du sujet et le nouveau par le prédicat qui correspond à la répartition indiquée des déterminatifs.

\section{Bilan}

La présente recherche concernant la relation entre la signification du nom et ses fonctions syntaxiques dans la phrase simple montre que le choix des formes grammaticales d'un nom dans ces fonctions syntaxiques n'est pas aléatoire, mais il est déterminé par les modèles de conception de la pensée, ce qui reflète une situation objective.

Notre analyse a montré que la signification lexicale impose des limites à la possibilité d'utiliser un nom dans certaines fonctions et structures syntaxiques. De son côté, la structure grammaticale modifie le contenu lexical du mot. Non seulement la construction syntaxique provient de la combinaison de mots, mais les mots sont souvent choisis pour une structure syntaxique particulière. Ainsi, la fonction syntaxique du sujet est dominée par les noms au singulier avec un déterminatif défini.

Certes, des langues modernes comme le français ont une souplesse, une aisance, une flexibilité importante. Le français se recommande notamment par son exactitude et sa clarté. Bien loin de tolérer les licences, les exagérations, les éclats approuvés par certaines langues voisines, il recherche en tout une précision telle qu'il n'admet rien, qui ait besoin de glose ou d'excuse.

L'analyse sémantique et grammaticale complète du nom en fonction des termes nominaux implique l'identification de la signification derrière cette forme. En deuxième étape de la recherche il est nécessaire de déterminer, par exemple, à quelle fréquence un nom au singulier est utilisé en fonction du sujet avec la valeur de l'unité, de la généralisation, etc.

\section{Bibliographic references}

ALISOVA, T. 2009. Essays on the syntax of the modern Italian language: semantic and grammatical structure of a simple sentence. Moscow: URSS. ISBN: 978-5-39700784-9.

BABUSHKINA, L.E. - KALUGINA, O.A. 2020. Students' media competence formation in French foreign language teaching in the practice of higher education, [Formation de la competence mediatique chez les etudiants en classe du francais langue etrangere dans la pratique de l'enseignement superieur]. In: XLinguae, vol. 13 n. 1, pp. 314-333. ISSN 1337-8384, eISSN 2453-711X. 
BALZAC, H. 2012. Le Père Goriot. New York: Hachette Book Group. ISBN: 9782012814684.

BALZAC, H. 2017. Eugenie Grandet. Moscow: T8. ISBN: 978-5-521-05379-7.

BAUGH, A.C. - CABLE, T. 2012. A History of the English Language. London: Routledge. ISBN-13: 978-0415655965.

COMBETTES, B. 2012. De quelques problèmes spécifiques à l'élaboration d'une grammaire historique. In: Langue française, n. 176, pp. 69-83. https://doi.org/10.3917/lf.176.0069.

DUBOIS, J. 1967. Grammaire structurale du français. Le verbe. Paris: Larousse. ISBN: 5-211-00391-8.

DUBOIS, J. - IRIGARAY L. 1966. Approche expérimentale des problèmes intéressant la production de la phrase noyau et ses constituants immédiats. In: Langages, vol. 1, pp 90-125, Available online: https://www.persee.fr/doc/lgge_0458726x_1966_num_1_3_2346. ISSN: 0458726X.

ETHEVES, C. 2016. Literary, Political And Cultural Stakes In The Textbooks Of French - Kaleidocope, Ecriture, Beaubourg And Rive Gauche Example: The Last Year Of The Italian High School [Enjeux Litteraires, Politiques Et Culturels Des Manuels De Français: Kaleidocope, Ecritures, Beaubourg Et Rive Gauche Exemple: Derniere Annee Du Lycee Italien]. In: Slavonic Pedagogical Studies Journal, vol. 5, n.1, pp.29-49. ISSN 1339-8660.

GAK, V. 2000. Theoretical grammar of the French language. Moscow: Dobrosvet. ISBN 5-7913-0035-2.

GAK, V. 2004. Conversations about the French word. Moscow: URSS. ISBN 5-35400797-6.

GREIMAS, A. 1996. Sémantique structurale. Recherche de méthode. Kirov: JSC House of Printing-Vyatka. ISBN 5-8291-0440-7.

LALINSKA, M. - STRANOVSKA, M. - TARDY, S. - ZIAK, P. 2020. Intervencny program citania s porozumenim pre francuzsky jazyk : uroven A2. Prague: Verbum, 2020. 198 p. ISBN 978-80-87800-71-3.

LAPTEVA, O. 1976. Russian colloquial syntax. Moscow: Nauka. ISBN: 9782221157831.

MARTINET, A. 1978. Structures élémentaires de l'énoncé. Paris: Versilio. ISBN: 2221127137.

MAURIAC, F. 2007. Le Noeud de viperes. Paris: Le Livre de Poche. ISBN: 978-2253-00287-1.

MAURIAC, F. 2009. Le Sagouin. Paris: Pocket. ISBN 978-2-266-02313-9

MAUROIS, A. 2014. Le cercle de famille. Paris: Grasset. ISBN: 2246146593, 9782246146599.

SAINT-EXUPERY, A. 2018. La Terre des Hommes. Paris: Gallimard. ISBN: 978-207-036021-5.

SHVEDOVA N. 1980. Grammar of the modern Russian literary language. Moscow: Nauka. ISBN-10: 2260017665.

VASILYEVA, N. M. 2010. French language. Theoretical grammar, morphology, syntax. Moscow: Yurayt Publishing House. ISBN: 978-5-534-06284-7.

ZOLA, E. 2002. L'Argent. Paris: Classiques universels. ISBN: 978-2-84595-065-8.

ZOLA, E. 2017. Pot-Bouille. Paris: Hatier. ISBN: 978-2-401-02823-4.

Words: 4671

Characters: 29594 (16,44 standard pages)

XLinguae, Volume 14 Issue 4, October 2021, ISSN 1337-8384, eISSN 2453-711X 
Assoc. Prof. Aleksei An. Zaitsev, PhD

Department of Russian and Foreign Languages

Russian State Agrarian University - Moscow Timiryazev Agricultural Academy

Timiryazevskaya Str., 49

127550 Moscow

Russia

a.zaizev@mail.ru

Assoc. Prof. Dinara G. Vasbieva, PhD

Department of English Language for Professional Communication

Financial University under the Government of the Russian Federation

Leningradsky prospect 49

125993 Moscow

Russia

dinara-va@list.ru 